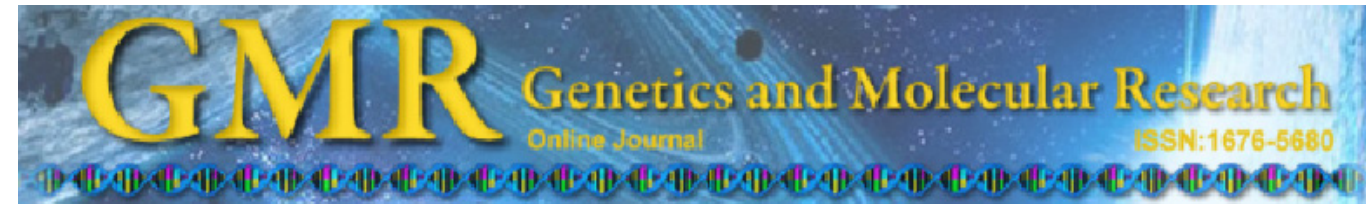

Review

\title{
Functional characterization and signal transduction ability of nucleotide-binding site-leucine-rich repeat resistance genes in plants
}

\author{
R.K. Joshi and S. Nayak \\ Centre of Biotechnology, Siksha O Anusandhan University, Bhubaneswar, India \\ Corresponding author: R.K. Joshi \\ E-mail: rajkumar.joshi@yahoo.com
}

Genet. Mol. Res. 10 (4): 2637-2652 (2011)

Received March 10, 2011

Accepted September 19, 2011

Published October 25, 2011

DOI http://dx.doi.org/10.4238/2011.October.25.10

\begin{abstract}
Pathogen infection in plants is often limited by a multifaceted defense response triggered by resistance genes. The most prevalent class of resistance proteins includes those that contain a nucleotide-binding site-leucine-rich repeat (NBS-LRR) domain. Over the past 15 years, more than 50 novel NBS-LRR class resistance genes have been isolated and characterized; they play a significant role in activating conserved defense-signaling networks. Recent molecular research on NBS-LRR resistance proteins and their signaling networks has the potential to broaden the use of resistance genes for disease control. Various transgenic approaches have been tested to broaden the disease resistance spectrum using NBS-LRR genes. This review highlights the recent progress in understanding the structure, function, signal transduction ability of NBS-LRR resistance genes in different host-pathogen systems and suggests new strategies for engineering pathogen resistance in crop plants.
\end{abstract}

Key words: R genes; NBS-LRR; Signal transduction 


\section{INTRODUCTION}

As plant cells do not have an immune system, they maintain a constant observation over the infecting pathogens by expressing large numbers of resistance $(R)$ genes. As per Flor's gene-for-gene interaction model, the induction of the plant defense response is initiated by the plant's recognition of specific molecules called elicitors encoded directly or indirectly by the avirulence alleles (avr) of the pathogens (Flor, 1971). Once this recognition has occurred, an intracellular signal transduction cascade is initiated, triggering activation of the defense arsenal of the challenged host plant cell (McDowell and Woffenden, 2003). Most R gene-triggered resistance is associated with a rapid defense response, termed the hypersensitive response (HR). The HR results in a localized cell and tissue death at the site of infection, which prevent the further spread of the infection (Hammond-Kosack and Jones, 1997). The local response, however, results in a non-specific systemic acquired resistance (SAR) throughout the plant.

A large number of plant resistance genes or elicitor receptors have been isolated and cloned based on positional or map-based cloning and transposon tagging (Sharma et al., 2009). The different isolated $\mathrm{R}$ genes have been grouped into five basic classes (Hammond-Kosack and Jones, 1997) (Figure 1). Among them, the genes that encode proteins containing a nucleotide-binding site (NBS) and C-terminal leucine-rich repeats (LRRs) represent the largest class of $\mathrm{R}$ genes. More than 50 different NBS-LRR R genes have been cloned and characterized from different plant species (Table 1). These cloned genes provide a lot of information about the structure and function of this class of $\mathrm{R}$ genes that exhibit resistance response against a variety of pathogens such as virus, bacteria, fungi, nematodes, and pests (Hulbert et al., 2001). Several different defense signaling components have been identified in NBS-LRR-mediated resistance signaling, which provide critical information on NBS-LRR gene-mediated defense response. The present review aims at discussing new developments in understanding the structure, function, genomic organization, and signal transduction mechanism of the NBS-LRR R genes in plants. The review also highlights the utility of NBS-LRR R genes for engineering pathogen resistance in crop plants.

\section{STRUCTURE AND FUNCTION OF NBS-LRR RESISTANCE GENES}

NBS-LRR class of gene encodes proteins with a variable N-terminal domain of approximately 200 amino acids, connected by a predicted NBS domain of approximately 300 amino acids (Traut, 1994) and a more variable C-terminal tandem array of approximately 10 to 40 short LRR motifs (Jones and Jones, 1997). In addition, the NBS-LRR genes are categorized into three subgroups based on the motif within their N-terminus: Toll/interleukin-1 receptor (TIR) group, coiled-coil (CC) or leucine zipper (LZ) group and non-motif group (Table 1).

\section{TIR AND NON-TIR DOMAINS}

The NBS-LRR class of $\mathrm{R}$ genes can be classified into two distinct subclasses based on the presence or absence of an N-terminal motif with homology to the cytoplasmic domains of the Drosophila Toll protein and the mammalian interleukin-1 receptor (TIR) (Pan et al., 2000). They are the TIR and non-TIR subclasses of NBS-LRR R genes, respectively. In recent articles, these two classes are referred to as 'CNL' and 'TNL' proteins, respectively (Meyers 
et al., 2005). These types of domains are believed to be involved in signaling and not ligand binding as they are found to have similarity with cytoplasmic signaling domains. TIR domain interactions between receptors and adaptors play a critical role in activating conserved cellular signal transduction pathways in response to bacterial lipopolysaccharides, microbial and viral pathogens and growth factors (Xu et al., 2000). Considering the similarity in sequence and related functions of the Drosophila, mammals and plant $\mathrm{R}$ genes, the plant proteins will function in an analogous manner. Moreover, the presence of TIR domains in plant $\mathrm{R}$ genes regulates the production of activated oxygen resulting in an oxidative burst during gene-for-gene defense responses (Hammond-Kosack and Jones, 1997). The TIR-type NBS-LRR genes occur extensively in dicots and are rare or absent in monocots (Bai et al., 2002; Meyers et al., 2003).

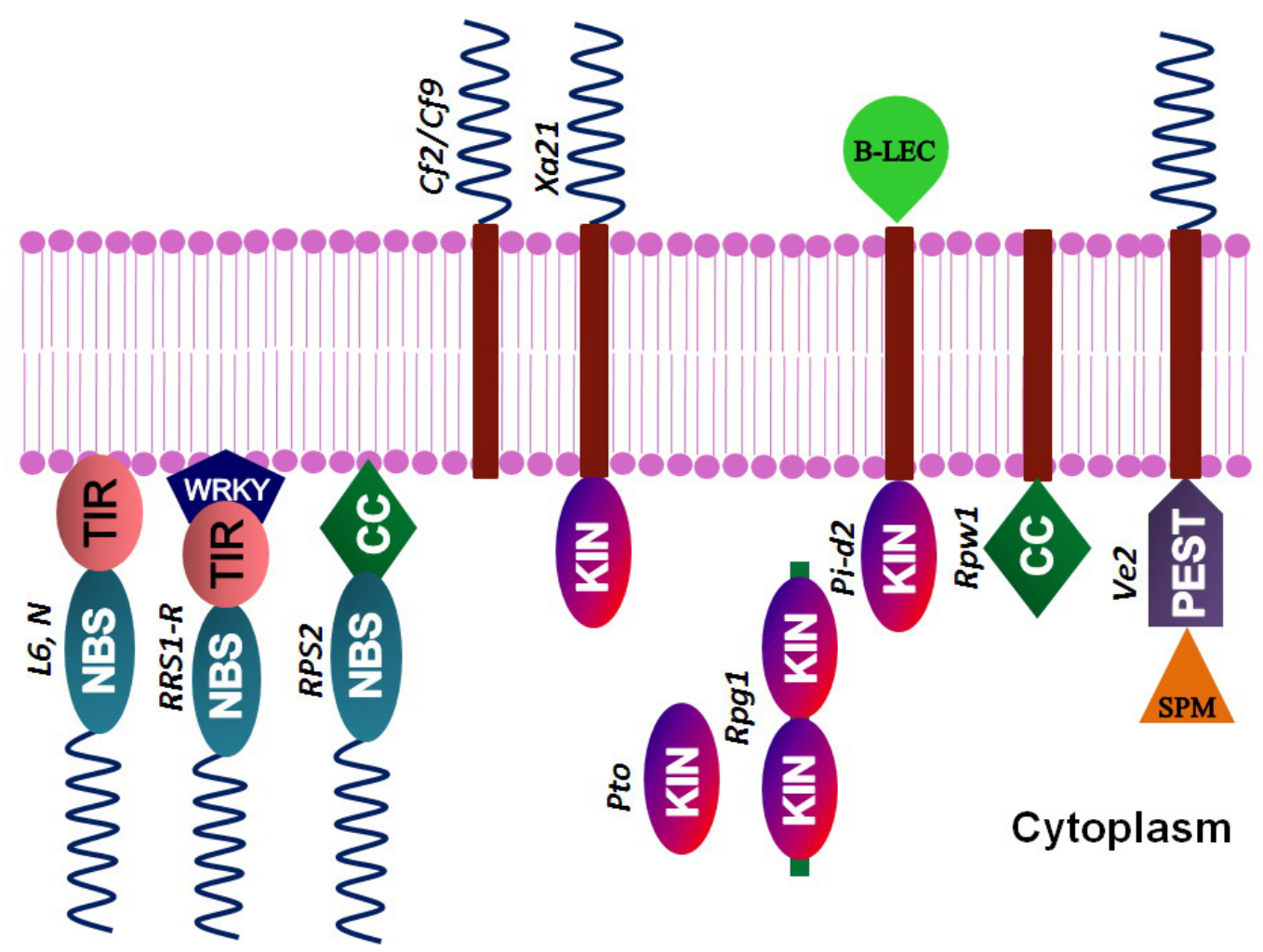

Figure 1. Major classes of $\mathrm{R}$ proteins. The widely represented R-protein family (NB-LRR) also contains a nucleotide-binding site (NBS). NB-LRRs are localized in cytoplasm as membrane proteins and contain either a TIR domain $(L 6, N)$ homologous to metazoan Toll-interleukin receptor or a putative coiled-coil (RPS2) domain at the N-terminus. A recent modification identified in the TIR-NBS-LRR protein is an additional transcriptional factor WRKY domain towards its C-terminus $(R R S 1-R)$. The class II family has extracellular LRRs attached with a transmembrane domain $(C f 2 / C f 9)$. The LRR kinase family contains extracytoplamic LRRs fused with cytoplasmic serine-threonine kinase $(\mathrm{KIN})$ domain $(\mathrm{Xa} 21)$. Another class includes only serine threonine kinase domain either single (Pto) or double ( Rpg1). A recently identified R gene (Pi-d2) has intracellular serine-threonine kinase attached with an extracellular binding-lectin (B-lectin). RPW8 (Rpw1) gene encodes a coiled-coil (CC) domain attached to a membrane anchor. A modified eLRR class gene has a transmembrane-LRR fused with a PEST domain for protein degradation $(\mathrm{Ve} 2)$. 
Table 1. Members of NBS-LRR disease resistance genes in plants.

\begin{tabular}{|c|c|c|c|}
\hline Gene & Host & Pathogen & Protein type \\
\hline RPS2 & Arabidopsis & Pseudomonas syringae & CC-NBS-LRR \\
\hline RPS5 & Arabidopsis & Pseudomonas syringae & LZ-NBS-LRR \\
\hline$R P M I$ & Arabidopsis & Pseudomonas syringae & CC-NBS-LRR \\
\hline$R P P 8 / H R T$ & Arabidopsis & Peronospora parasitica & CC-NBS-LRR \\
\hline$R P P 13$ & Arabidopsis & Peronospora parasitica & CC-NBS-LRR \\
\hline$R C Y 1$ & Arabidopsis & Cucumber mosaic virus & CC-NBS-LRR \\
\hline$R P P / H R T$ & Arabidopsis & Turnip crinkle virus & CC-NBS-LRR \\
\hline$R P M I$ & Tomato & Pseudomonas syringae & CC-NBS-LRR \\
\hline $\operatorname{Prf}$ & Tomato & Pseudomonas syringae & CC-NBS-LRR \\
\hline 12 & Tomato & Fusarium oxysporum & CC-NBS-LRR \\
\hline$M i-1$ & Tomato & Meloidogyne javanica & CC-NBS-LRR \\
\hline$M i-9$ & Tomato & Meloidogyne javanica & CC-NBS-LRR \\
\hline$S w-5 / M i$ & Tomato & Tospovirus & CC-NBS-LRR \\
\hline$R \times 2$ & Potato & PVX (Potato X virus) & CC-NBS-LRR \\
\hline Gpa2/RxI & Potato & Globodera pallida/PVX & CC-NBS-LRR \\
\hline RI & Potato & Phytophthora infestans & CC-NBS-LRR \\
\hline Mlal & Barely & Blumeria graminis & CC-NBS-LRR \\
\hline Mla6 & Barely & Blumeria graminis & CC-NBS-LRR \\
\hline Mla12 & Barely & Blumeria graminis & CC-NBS-LRR \\
\hline Mla13 & Barely & Blumeria graminis & CC-NBS-LRR \\
\hline$P i b$ & Rice & Magnaporthe grisea & CC-NBS-LRR \\
\hline$P i-t a$ & Rice & Magnaporthe grisea & CC-NBS-LRR \\
\hline Pi36 & Rice & Magnaporthe grisea & CC-NBS-LRR \\
\hline$X a 1$ & Rice & Xanthomona oryzae & CC-NBS-LRR \\
\hline Rpl & Maize & Puccinia sorghi & CC-NBS-LRR \\
\hline$D m 3$ & Lettuce & Bremia lactucae & CC-NBS-LRR \\
\hline Bs2 & Pepper & Xanthomonas campestris & CC-NBS-LRR \\
\hline$P m 3 b$ & Wheat & Blumeria graminis & CC-NBS-LRR \\
\hline Lr10 & Wheat & Puccinia triticina & CC-NBS-LRR \\
\hline Pl8 & Sunflower & Plasmopara halstedii & CC-NBS-LRR \\
\hline$R B$ & Potato & Phytophthora infestans & CC-NBS-LRR \\
\hline Rpi-blbl & Potato & Phytophthora infestans & CC-NBS-LRR \\
\hline Fom-2 & Melon & Fusarium oxysporum & CC-NBS-LRR \\
\hline Rpgl-b & Soybean & Pseudomonas syringae & CC-NBS-LRR \\
\hline$R 3 a$ & Potato & Phytophthora infestans & CC-NBS-LRR \\
\hline Rxol & Maize & Xanthomonas oryzae & CC-NBS-LRR \\
\hline RPS4 & Arabidopsis & Pseudomonas syringae & TIR-NBS-LRR \\
\hline$R P P I$ & Arabidopsis & Peronospora parasitica & TIR-NBS-LRR \\
\hline Rpp10 & Arabidopsis & Peronospora parasitica & TIR-NBS-LRR \\
\hline Rpp14 & Arabidopsis & Peronospora parasitica & TIR-NBS-LRR \\
\hline$R P P 4$ & Arabidopsis & Peronospora parasitica & TIR-NBS-LRR \\
\hline RPP5 & Arabidopsis & Peronospora parasitica & TIR-NBS-LRR \\
\hline SSI4 & Arabidopsis & P. syringae pv. maculicola & TIR-NBS-LRR \\
\hline$R L M$ & Arabidopsis & Leptosphaeria maculans & TIR-NBS-LRR \\
\hline L6 & Flux & Melampsora lini & TIR-NBS-LRR \\
\hline$L$ & Flux & Melampsora lini & TIR-NBS-LRR \\
\hline$M$ & Flux & Melampsora lini & TIR-NBS-LRR \\
\hline$P$ & Flux & Melampsora lini & TIR-NBS-LRR \\
\hline$P 2$ & Flux & Melampsora lini & TIR-NBS-LRR \\
\hline Bs4 & Tomato & Xanthomonas campestris & TIR-NBS-LRR \\
\hline$N$ & Tobacco & Tobacco mosaic virus & TIR-NBS-LRR \\
\hline$R R S 1-R$ & Arabidopsis & Ralstonia solanacearum & $\begin{array}{l}\text { WRKY-TIR- } \\
\text { NBS-LRR }\end{array}$ \\
\hline$P i-9$ & Rice & Magnaporthe grisea & NBS-LRR \\
\hline $\mathrm{Pi2}$ & Rice & Magnaporthe grisea & NBS-LRR \\
\hline Piz- $t$ & Rice & Magnaporthe grisea & NBS-LRR \\
\hline Cre3 & Wheat & Heterodera avenuae & NBS-LRR \\
\hline Crel & Wheat & Heterodera avenuae & NBS-LRR \\
\hline$I 2 C$ & Tomato & Fusarium oxysporum & NBS-LRR \\
\hline Hero & Tomato & Globodera rostochiensis & NBS-LRR \\
\hline
\end{tabular}

$\mathrm{NBS}=$ nucleotide-binding site; $\mathrm{LRR}=$ leucine-rich repeat $\mathrm{CC}=$ coiled-coil structure; $\mathrm{LZ}=$ leucine zipper regions; $\mathrm{TIR}=$ Toll/interleukin-1 receptor; WRKY $=$ DNA binding domain . 
The non-TIR subclass is basically characterized by the presence of a CC structure or an LZ structure (Pan et al., 2000; Hulbert et al., 2001). The CC structure consists of two or more $\alpha$-helices with a super-helical twist and represents a heptad repeat sequence (bcdefg), where ' $\mathrm{a}$ ' and ' $\mathrm{d}$ ' are hydrophobic residues, while the residues at the 'e' and ' $\mathrm{g}$ ' positions are charged and polar. Most of the NBS-LRR genes in dicots and monocots including cereals contain the $\mathrm{CC}$ structure. It is believed that the $\mathrm{CC}$ or $\mathrm{LZ}$ domain facilitates the formation of a $\mathrm{CC}$ structure to promote oligodimerization with a wide variety of proteins including structural proteins and transcription factors or specific interactions with other proteins (Burkhard et al., 2001). $\mathrm{R}$-gene products can exist as monomers before infection and then undergo dimerization upon activation or they can even exist initially as a dimer before pathogen challenge and break into monomers after activation. However, its actual role in $\mathrm{R}$ gene function is still unknown. It remains unclear whether any protein can indeed interact with R-gene products through LZ regions or R-gene products can really undergo dimerization through LZ regions. Recently, it has been found that the LZ domain of the RPM1 gene interacts with RPM1 interacting protein RIN4 to negatively regulate resistance to Pseudomonas syringae (Mackey et al., 2002).

\section{NUCLEOTIDE BINDING SITE DOMAIN}

The majority of $\mathrm{R}$ genes are characterized by amino acid sequences with strong similarity to NBSs. This NBS domain occurs in diverse proteins in addition to R genes with ATP or GTP binding activity such as ATP synthase $b$ subunits, ras proteins, ribosomal elongation factors, adenylate kinase, etc. (Traut, 1994). A typical NBS domain of resistance R gene broadly consists of the generalized consensus $\operatorname{Gxxx\mathrm {xK}}(\mathrm{T} / \mathrm{S})$ for the kinase 1a, phosphate-binding loop (P-loop) followed by a kinase 2 domain with four consecutive hydrophobic amino acids followed by a conserved aspartate, which coordinates the metal ion binding required for phospho-transfer reactions. The subsequent kinase 3 a motif contains a conserved tyrosine (Y) or arginine (R) amino acid and interacts with the purine base of ATP (Traut, 1994). The NBS domains of $\mathrm{R}$ genes are highly homologous to NBS regions of apoptosis-related genes like CED4 from Caenorhabditis elegans and APAF-1, FLASH and Nod1 from humans (Van der Beizen et al., 1998), which facilitates NTP binding. Therefore, the NBS domain plays a significant role in plant defense signaling. The presence of an NBS domain in R genes suggests possible activation of a kinase or $\mathrm{G}$ proteins in the resistance response. The tomato $I-2$ and Mi-1 genes are found to have ATP binding ability at the P-loop site of the kinase 1a domain (Tameling et al., 2002). It is believed that nucleotide triphosphate binding is essential for the functioning of R-gene products because site-specific mutations in the NBS regions have resulted in loss or negative effect of function in different $\mathrm{R}$ genes such as RPS2 of Arabidopsis and $N$ gene of Tobacco.

The TIR and non-TIR subclasses of NBS-LRR genes can also be distinguished by the motifs found within the NBS domain. The last amino acid residue in the NBS kinase 2 motif is generally an aspartic acid (D) or aspartate (N) in the TIR subclass and tryptophan (W) in cases of non-TIR subclass (Pan et al., 2000). The TIR-NBS types are largely distributed in dicots while the non-TIR-NBS types are often found in both monocots and dicots. The conserved P-loop (GVGKTT) and GLPLA hydrophobic motifs of the NBS domain (Figure 2) are often used in rapid isolation of the NBS-LRR genes or resistance gene candidates (RGCs) from different plant species by using a polymerase chain reaction (PCR)-based approach with degen- 
erate oligonucleotide primers. Genetic mapping and phylogenetic analysis have revealed that many of the RGCs either co-segregate with or are closely linked to known disease resistance loci (Kanazin et al., 1996; Mago et al., 1999; Pan et al., 2000) suggesting that these NBS sequences may form part of the R genes. Hence, the NBS domain is often used in the identification and classification of $\mathrm{R}$ genes as they not only recognize R-gene sequences in the available nucleotide databases but also exhibit great alignment with R-gene sequences at the conserved motifs and classify NBS-LRR genes as TIR or non-TIR types (Bai et al., 2002).

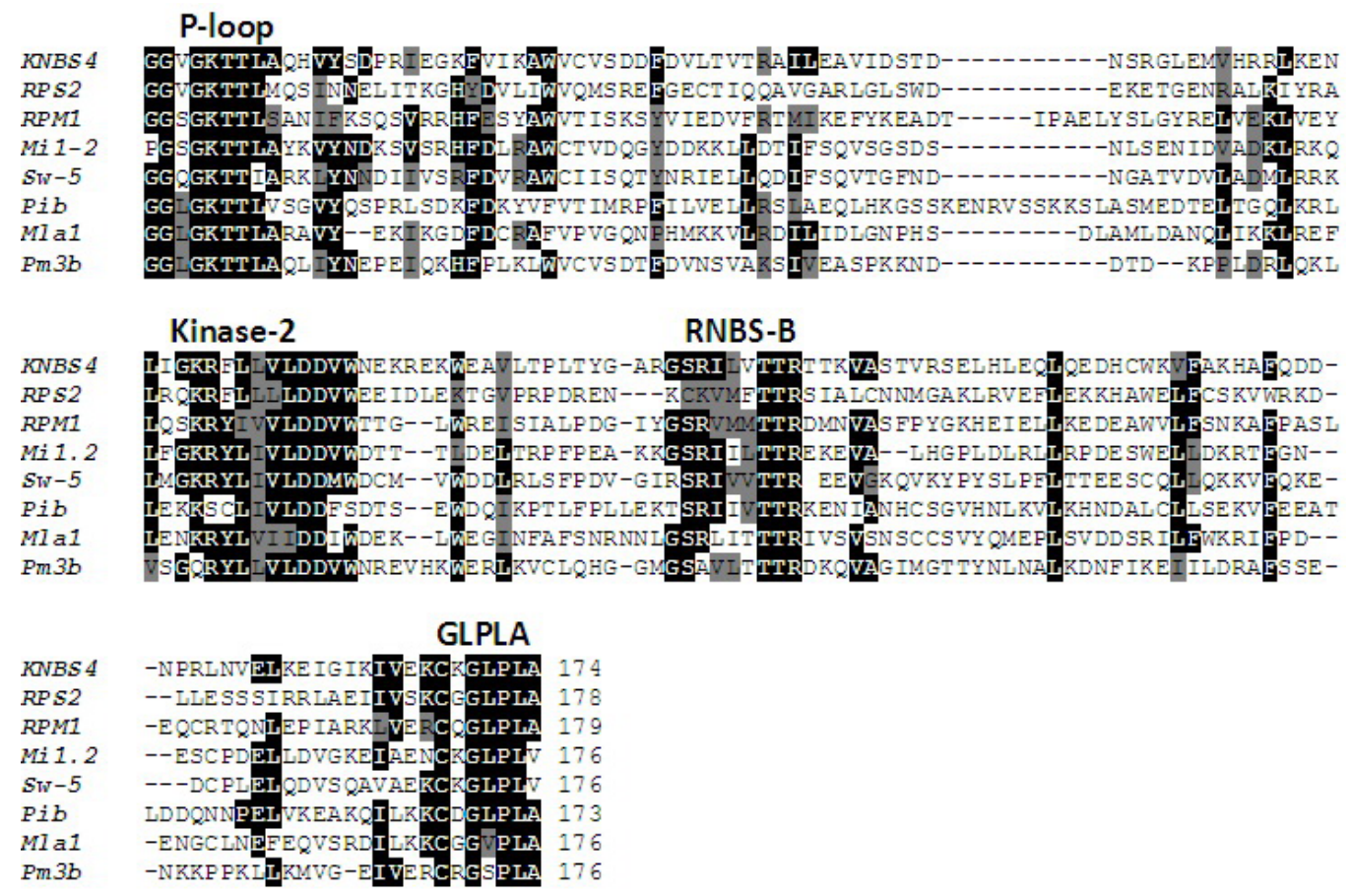

Figure 2. Alignment of the NBS domain of multiple NBS-LRR disease resistance proteins. The conserved NBS domains as determined by Meyers et al. (1999) are indicated. Identical amino acids are shaded in black and conservative substitutions are shaded in gray. $\mathrm{NBS}=$ nucleotide-binding site; $\mathrm{LRR}=$ leucine-rich repeat.

\section{LEUCINE-RICH REPEATS}

The LRR domain consists of tandemly arranged repeats in the C-terminus of the NBSLRR genes. It basically consists of 20-29 amino acid residues with a conserved 11 residue sequence $(\operatorname{Lxx} \operatorname{Lx} \operatorname{Lxx}(\mathrm{N} / \mathrm{C} / \mathrm{T}) \mathrm{xL})$, where $x$ is any residue and $L$ can be replaced with valine or isoleucine (Kobe and Kajava, 2001). A distinct LRR contains a tertiary structure resembling a curved spring with each curl representing a single LRR. The porcine RNase inhibitor contains LRR of 29 amino acids while it is believed that an LRR domain with an average length of 24 amino acids will resemble a $\beta$-helical array (Yoder et al., 1993). The exposed amino acids of LRRs determine its functional specificity while the hydrophobic residues determine the struc- 
ture. In some R-gene products, a short stretch of amino acids designated $\mathrm{C} 2$ that divides the LRR region into amino terminal LRRs (C1) and carboxyl terminal LRRs (C3). It is believed that this short "loop out" domain not only acts as a molecular hinge but also interacts with other proteins that participate in signal transduction (Hammond-Kosack and Jones, 1997). Often, the LRR regions are flanked by several cysteine residues for maintaining the overall protein structure.

There are as many as seven distinct LRR subfamilies, which provide structural framework to protein interactions in various cellular processes such as interaction between enzymes and enzyme inhibitors, binding of peptide hormones by transmembrane receptors, etc. (Torii, 2004). The LRR domains are not only found in NBS-type R genes but also in other types of R genes like the LRR-TM (transmembrane) and LRR-PK (protein kinase). However, except for NBS-LRR genes, the LRR domains are located outside the cell and N-terminal in nature. It has been found that the LRR domain of many R-gene products acts as a binding domain for the ligand (elicitor) produced due to the Avr gene activity. For an extracytoplasmic predicted R-gene product with the LRR motif and the domain being well conserved, there is a possibility that the hydrophobic face can facilitate multiple interactions with other ligands (HammondKosack and Jones, 1997). Zhang et al. (1995) have previously shown that the glycosylation pattern within the LRR domain is directly involved in ligand binding. It has been found that the glycosylation sites are generally absent in the $\beta$-strand of the LRR region of the $\mathrm{C} 1$ domain and so the hydrophobic end plays a role in the interaction, while the glycosylation sites are present within the $\beta$-strand of $\mathrm{C} 3$ leucine repeats. This makes it essential to study the crystal structure of various plant LRR proteins. In other words, LRRs directly facilitate the interaction of R-gene products with proteins involved in defense signal response and by default accepted as the recognition domain because all the other motifs exhibit signaling capacity. Moreover, related evidence also suggests that LRRs are major determinants of resistance specificity (Hulbert et al., 2001). Jia et al. (2000) have shown that the resistance specificity of the Pi-ta gene against rice blast varies with a single amino acid difference in the LRR domain. Likewise, six amino acid changes between the flax rust resistance genes $P$ and $P 2$ within the $\beta$-strand of four LRR units determine resistance specificity (Dodds et al., 2001). However, in recent times, it has also been found that the LRR domain can also interact with other domain of $\mathrm{R}$ proteins to block the activation of resistance signaling (Hammond-Kosack and Kanyuka, 2007). Ade et al. (2007) have shown that the LRR domain interacts with the NBS domain resulting in inactivation of defense signaling of the RPS5 gene in transgenic tobacco. However, further investigation is needed to validate this observation in other types of $\mathrm{R}$ genes.

\section{ORGANIZATION OF NBS-LRR GENES IN THE PLANT GENOME}

NBS-LRR genes are abundant in plant genomes. Meyers et al. (2003) characterized the complete set of plant R gene-related NBS-encoding genes in the Col-0 Arabidopsis genome. Bioinformatics analysis together with experimental validation have identified more than 160 NBS-LRR-encoding genes. It included the 11 cloned $\mathrm{R}$ genes or the closest Col-0 homologs to R genes cloned from other Arabidopsis ecotypes. Fifty-eight additional Arabidopsis genes were identified, which encoded TIR motifs but no LRRs. The NBS-LRR genes were evenly distributed throughout the genome with chromosome I containing 49 genes; chromosome II, 4 genes; chromosome III, 18 genes; chromosome IV, 31 genes, and chromosome 
V, 59 genes. Forty-six Arabidopsis R genes were singletons, 25 were doublets, 7 were triplets, and many individual loci with four, five, seven, and nine copies. Approximately two-thirds of these genes also encode a "Toll-interleukin homology" domain at the N-terminus of the predicted protein (the "TIR" class), while the remaining one-third encode a CC motif at the N-terminus (the "non-TIR" class). Phylogenetic analysis demonstrates that known resistance genes are present in most of the major clades. Classification based on protein domains, intron positions, sequence conservation, and genome distribution defined four sub-groups of CC-NBS-LRR proteins, eight subgroups of TIR-NBS-LRR proteins and an additional pair of divergent NBS-LRR proteins that lacked a defined N-terminal motif.

In rice, Bai et al. (2002) detected 250 predicted full-length NBS-LRR genes and 560 NBS sequences, but these numbers do not accurately reflect those in any given rice genome, mainly because they are based on the analysis of incomplete databases that contain sequences from japonica rice and indica rice. Zhou et al. (2004) surveyed the NBS-encoding genes in the complete genome sequence of one japonica cultivar, Oryza sativa L. var. Nipponbare and found 535 NBS-coding sequences, including 480 non-TIR NBS-LRR genes. TIR-NBS-LRR genes, which are common in A. thaliana, have not been identified in the rice genome. A few genes with the TIR have been identified in rice, but these did not encode any obvious LRR domain, and were otherwise divergent from NBS-LRR genes (Bai et al., 2002). The number of non-TIR NBS-LRR genes in rice is 8.7 times higher than that in $A$. thaliana, and they account for about $1 \%$ of all predicted ORFs in the rice genome. The chromosomal distribution of the NBS-encoding genes in rice genome is significantly non-random. This can be known from the fact that chromosome 9 contains only 20 NBS genes, while chromosome 11 contains 133 of the total number of NBS genes, 263 genes (51\%) resided in 44 gene clusters, and that the average number of genes in a cluster was six. There are 15 clusters with four copies, 13 with five, 4 with six, 4 with seven, 3 with eight, 3 with ten, and 1 each with 15 or 17 NBS-coding genes. Besides these clusters, there were 40 tightly linked doublets and 17 triplets. Therefore, a total of 394 genes resided either in a gene cluster or in a tandem array. In all, 125 NBS singletons were dispersed over all the chromosomes. The ratio of singletons to the total number of NBS genes in the rice genome (24.1\%) was similar to that in Arabidopsis (26.8\%; Meyers et al., 2003).

Kohler et al. (2008) examined the diversity and distribution of NBS-LRR genes in Populus trichocarpa (a model tree species) draft genome sequence.

The NBS class of genes in this perennial tree is large and diverse, comprised of $\sim 400$ genes, at least twice the complement of Arabidopsis. The NBS family can be divided into multiple subfamilies with distinct domain organizations. It includes 119 CC-NBS-LRR genes, 64 TIR-NBS-LRR genes, 34 BED-finger-NBS-LRR, and both truncated and unusual NBS and NBS-LRR-containing genes. Similarly, Mun et al. (2009) used the genome sequence of Brassica rapa to identify NBS-encoding genes in the Brassica genome. They identified 92 non-redundant NBS-encoding genes [30 CC-NBS-LRR (CNL) and 62 TIR-NBS-LRR (TNL) genes] in approximately $100 \mathrm{Mbp}$ B. rapa euchromatic genome sequence. Despite the fact that $B$. rapa has a significantly larger genome than $A$. thaliana due to a recent whole genome triplication event after speciation, $B$. rapa contains a relatively small number of NBS-encoding genes compared to $A$. thaliana, presumably because of deletion of redundant genes related to genome diploidization.

Thus, it appears that many R genes are tightly linked in clusters within plant genomes. Hulbert et al. (2001) reviewed this phenomenon and noted that genetically linked alleles or 
clusters of genes have a greater possibility for recombination than simple loci composed of single genes. Such clustering of R genes may also contribute to a generation of novel resistance alleles through recombination and gene conversion.

\section{EXPRESSION OF NBS-LRR GENES}

Although the functional role of NBS-LRRs is devoted to defense response, the level of expression of $\mathrm{R}$ genes is an area that is still under discussion due to its low transcript levels (Graham et al., 2000) and only a few reports have been published in the literature on R gene expression. The results on expression on many $\mathrm{R}$ genes have demonstrated that they are expressed constitutively at a very low level, before pathogen challenge (Hammond-Kosack and Jones, 1997; Hulbert et al., 2001), or are induced following pathogen inoculation (Yoshimura et al., 1998). Northern or RT-PCR analyses on different R genes have revealed the presence of low levels of transcripts in plants that have not been infected with a pathogen (Parker et al., 1997; Mes et al., 2000). These findings are in agreement with the postulated role of NBS-LRR proteins acting as preformed receptors that recognize a pathogen in a gene-for-gene correlation for disease and resistance in plants (Van der Biezen and Jones, 1998). Likewise, there is induced expression of transcript of the rice $\mathrm{Xa} 21$ resistance gene, with pathogen inoculation (Yoshimura et al., 1998). Another exception is the recently isolated rice $\mathrm{Xa} 27$ gene, which is differentially expressed in the presence of AvrXa27 bacterial effector. The same is true for the expression of the resistance gene candidates. While studying RGC expression, 11 of 29 RGCs in Brassica (Fourmann et al., 2001) and 10 of 15 RGCs in Zingiber officinale (Nair and Thomas, 2007) were expressed. The lack of a circulatory system in plants might be compensated for by a capacity to express each $\mathrm{R}$ protein constitutively in every cell that potentially could be attacked. However, the expression of single $\mathrm{R}$ proteins is not required for life even in environments where the pathogen thrives. When $\mathrm{R}$ genes are expressed from their native promoter in taxonomically unrelated species or over-expressed in their native plant species this frequently leads to precocious defense activation and plant cell death (Hammond-Kosack and Kanyuka, 2007).

It is also believed that there are fitness costs associated with the expression of R genes and activation of defense response pathways in absence of a pathogen. Multiple mutation at the RPP5 locus in Arabidopsis results in enhanced disease resistance due to constitutive upregulation of defense responses. Yi and Richard (2007) analyzed the regulation of gene expression at the RPP5 locus in the Arabidopsis Col-0 accession and found that multiple $\mathrm{R}$ genes are coordinately regulated at this locus through both transcriptional activation and RNA-silencing mechanisms. This feedback mechanism greatly contributes to the optimization of defense response not only by reducing the fitness costs but also by enhancing the capacity for induction of defense responses with pathogen attack.

\section{NBS-LRR SIGNAL TRANSDUCTION}

Physical interaction between $\mathrm{R}$ proteins and pathogen effectors forms the basis of the gene-for-gene model and results in the plant defense responses and eventually leads to resistance (Keen, 1990). The tomato non-NBS-LRR Pto protein kinase interacts directly with its cognate bacterial effector AvrPto within the serine/threonine kinase activation domain at residue threonine 204. Tomato Pto also interacts directly with a second bacterial effector AvrPtoB, 
which has intrinsic E3 ubiquitin ligase activity. This direct interaction with effectors has also been demonstrated in NBS-LRR genes. The Arabidopsis RRS1-R protein interacts with the bacterial type III effector Pop2 (Deslandes et al., 2003), rice Pi-ta interacts with AVR-Pita (a predicted secreted metalloprotease) from the Ascomycete rice blast fungus Magnaporthe grisea (Jia et al., 2000), while the flax L5, L6 and L7 proteins interact in yeast with the corresponding AvrL567 protein variants from the Basidiomycete flax rust fungus Melampsora lini (Dodds et al., 2001). However, lack of evidence on direct R-Avr interactions in plants or in vitro created the assumption that various alternative recognition targets and even multi-protein recognition complexes also exist. This has led to the guard hypothesis, which predicts that an effector protein interacts with a host target, which is itself recognized by more than one $\mathrm{R}$ protein (Dangl and Jones, 2001). The Arabidopsis RIN4 protein is an example of a host target for type III bacterial effectors, which is recognized by at least two CNL R proteins (Mackey et al., 2002). RIN4 is a 211-amino acid acetylated protein in the plasma membrane, which is targeted by structurally unrelated bacterial effectors AvrRpm1 and AvrB. Both effectors induce phosphorylation of RIN4, which activate the R protein RPM1. A third effector AvrRpt2 is recognized by RIN4 inside the plant cell, which cleaves RIN4 at two sites. Cleavage of RIN4 activates the NB-LRR protein, RPS2.

Activation of HR on pathogen inoculation triggers a resistance response called SAR. This results in the accumulation of salicylic acid (SA) throughout the plant and the consequent expression of a characteristic set of defense genes (Figure 3). Plants expressing SAR are more resistant to subsequent attack by a variety of other pathogens (Glazebrook, 2001). Some defense responses are activated by signal transduction networks that require jasmonic acid (JA) and ethylene (ET) as signal molecules. The discovery of new genes or mutants allows further dissection of local and systemic signaling networks and begins to highlight the complex interplay between defense molecules such as SA, nitric oxide, reactive oxygen intermediates, JA and ET (Thomma et al., 2001). Feys and Parker (2000) detected two mutants' $n d r l$ and $e d s 1$ that suppress race-specific resistance to strains of the bacterium Pseudomonas syringae. EDS1 encodes a lipase-like protein while NDR1 encodes a membrane-associated protein, and they are independently required for the function of different NBS-LRR genes. The R genes suppressed by the $n d r 1$ mutation are not affected by $e d s 1$ mutants and vice versa. $E d s 1$ suppresses TIR-NBS-LRR R genes, whereas $n d r l$ suppresses a subset of non-TIR-NBS-LRR R proteins. Recent studies have revealed that $\mathrm{R}$ gene-mediated resistance is regulated by the cytoplasmically localized signaling complex SGT1/RAR1/HSP90 [SGT1 (suppressor of G2 allele of SKP1), RAR1 (required for Mla-dependent resistance 1) and HSP90 (heat shock protein 90)] in plants as diverse as Arabidopsis, barley and tobacco (Azevedo et al., 2002; Muskett et al., 2002). Proteins in this complex work together to stabilize various NBS-LRR R proteins. RAR1 encodes a small zinc-binding protein that interacts with SGT1 in barley and tobacco extracts. The SGT1 component is also essential for the function of Skp1-Cullin-F-box protein (SCF) E3 ubiquitin ligase complex, which targets proteins for degradation by the 26S proteasome. The ubiquitin-proteasome pathway is therefore likely to be an important modulator of $\mathrm{R}$ gene-triggered resistance.

Other important defense regulators to emerge are components of mitogen-activated protein kinase (MAPK) cascades that constitute functionally conserved eukaryotic signal relay systems in response to various environmental stresses (Figure 3) (Asai et al., 2002). Importantly, the MAPK kinase, EDR1, negatively regulates SA-inducible defenses (Frye et 


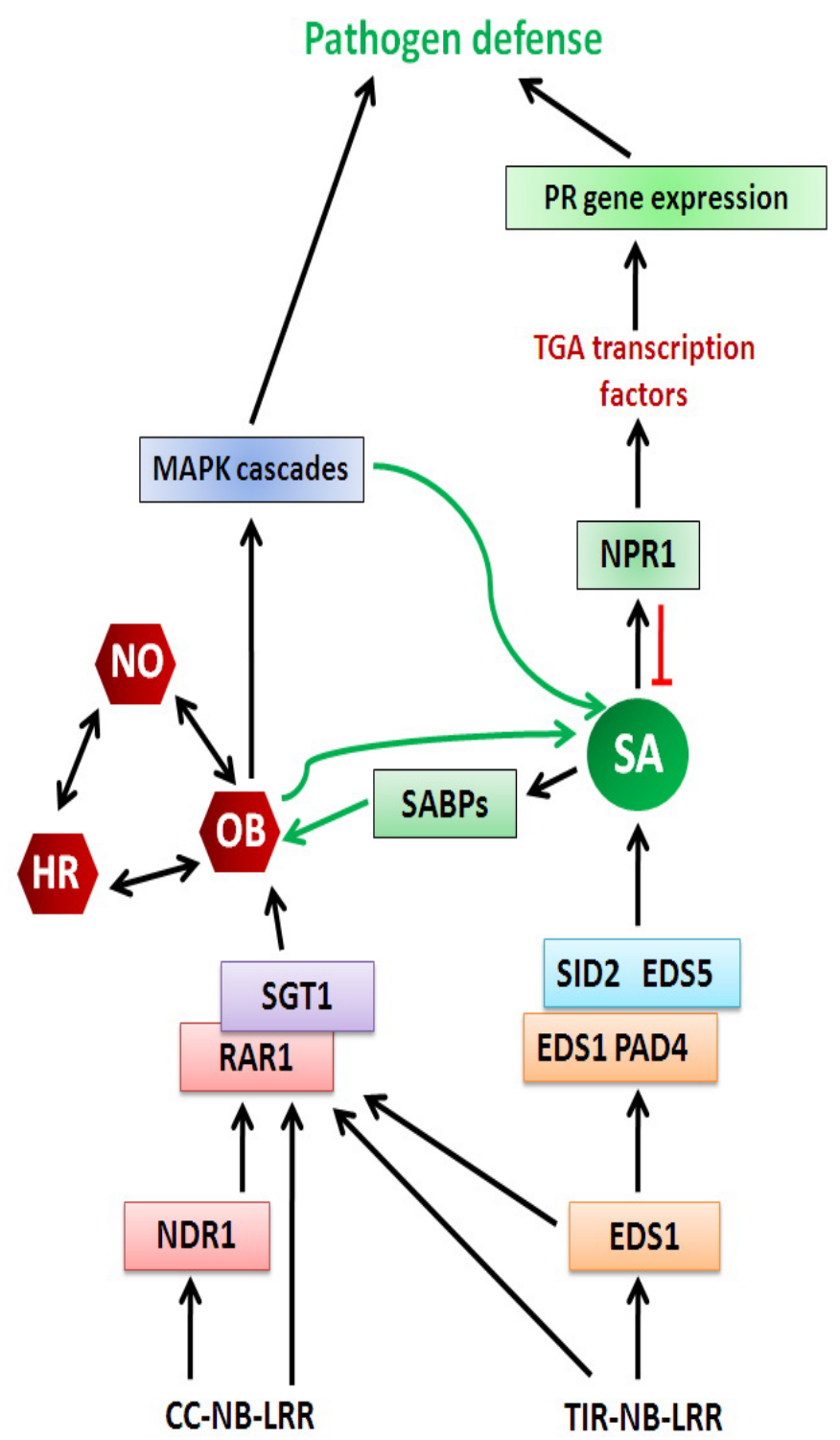

Figure 3. R protein-dependent activated signaling cascades for CC-NBS-LRR and TIR-NBS-LRRs. CC-NBSLRR-type R proteins require NDR1 (non-race-specific disease resistance 1) whereas TIR-NBS-LRRs depend on EDS1 (enhanced disease susceptibility 1). Both the pathways converge at RAR1/SGT1 operating upstream of hypersensitive response (HR) and oxidative burst (OB). Various salicylic acid (SA)-binding proteins (SABP) located in distinct cellular compartments may modulate the local concentrations of available SA signal. The OB can potentiate SA-mediated signaling directly and via the induction of various MAPK cascades in case of CC-NBSLRRs. Activation of oxidative burst potentiating defense responses of TIR-NBS-LRR proteins involves combined actions of EDS1 and PAD4 (phytoalexin deficient 4), EDS5, SA and NPR1 (non-expressor of PR1). NPR1 is required downstream from SA, which also stimulates NPR1 translocation into the nucleus where it interacts with TGA (TGACG DNA motif) transcription factors and induces the expression of PR genes. $\mathrm{NO}=$ nitric oxide; MAPK $=$ mitogen-activated protein kinase. For other abbreviation, see legend to Table 1 . 
al., 2001) whereas MAPK 4 appears to differentially regulate SA and JA signals (Petersen et al., 2000). These findings strongly implicate MAPK modules in molecular communication between different plant defense pathways. Another important feature of the systemic signaling is the ankyrin repeat protein, NPR1, initially identified as an SA response regulator, is required for both SAR and ISR (induced systemic resistance). The addition of SA to Arabidopsis seedlings promotes movement of NPR1 to the nucleus where it is able to bind several TGA (TGACG DNA motif) class transcription factors, conferring a possible direct route to defense gene induction (Fan and Dong, 2002). Identification of an apoplastic lipid transfer protein, DIR1, as an inducer of long distance defense signaling in SAR suggests that lipid-derived molecules may also play a role (Maldonado et al., 2002).

\section{PATHOGEN RESISTANCE IN CROP PLANTS USING NBS-LRR GENES}

$\mathrm{R}$ gene-mediated resistance has several attractive features for disease control. When induced in a timely manner, the concerted responses can effectively halt pathogen growth with minimal collateral damage to the plant (McDowell and Woffenden, 2003). Conventional plant breeding has significantly improved the resistance of crops to important diseases. However, they are time-consuming lengthy programs and create difficulties when trying to respond to the spread of new virulent pathogen strains. In recent times, DNA marker technologies have overcome such limitations. Markers are now used by leading breeding companies in markerassisted selection (MAS) programs for the rapid and effective transfer of $\mathrm{R}$ genes and other useful traits into elite crop breeding lines. However, MAS is expensive, which currently restricts its use. This has been simplified by simultaneous introduction of several $\mathrm{R}$ genes targeting different races of the same pathogen into one elite breeding line through the process of gene pyramiding. In the recent past, techniques for routine accurate detection of single nucleotide polymorphisms have emerged. These techniques have permitted the development of a novel method, called TILLING (targeting induced local lesions in genomes) for the discovery of allelic series of point mutations for any gene of interest in populations of chemically induced mutants (McCallum et al., 2000). By TILLING genotypes bearing different variant alleles of R gene, effector target, defense signaling gene, or defense response gene can be identified. These genotypes can then be evaluated for improved resistance to specific pathogens, followed by rapid introgression of the best alleles either singly or in combination into elite breeding materials.

Currently, the availability of cloned $\mathrm{R}$ genes for genetic transformation is opening the possibility of direct transfer into elite lines within a single generation. By plant transformation, several different $\mathrm{R}$ gene alleles, each effective against a single pathogen species or race, can be simultaneously introduced into semi-elite and elite germplasm. In theory, this should slow down the process of pathogen evolution, because for a virulent pathogen to emerge this would require all the corresponding Avr genes to mutate simultaneously within a single pathogen isolate (Hammond-Kosack and Kanyuka, 2007). Also, by taking the plant transformation route, the interspecies infertility barrier is removed; therefore, a wider source of useful resistance gene sequences is available. For example, different $\mathrm{R}$ genes have been introduced by direct gene transfer into heterologous species and have shown to confer resistance to pathogens carrying the corresponding Avr genes in the Solanaceae family (Thilmony et al., 1995; Tai et al., 1999). However, there has been no successful demonstration that $\mathrm{R}$ genes confer disease 
resistance when transferred to plants from a different family. For example, the R gene $B s 2$ expresses in pepper and the corresponding gene $A v r B s 2$ induces cell death in various heterologous species of Solanaceae but not in Arabidopsis. Likewise the Arabidopsis RPS2 gene that confers resistance to $P$. syringae is non-functional in the tomato. This phenomenon is often termed as 'restricted taxonomic functionality' (Tai et al., 1999). However, this concept is not a general attribute and Xiao et al. (2003) have demonstrated that the R genes RPW8.1 and $R P W 8.2$ from $A$. thaliana, a member of the family Brassicaceae, conferred resistance to powdery mildew diseases of $N$. tabacum and $N$. benthamiana, members of the family Solanaceae.

The rapid activation of localized defense responses that often results in hypersensitive reaction is one of the most prevalent and effective mechanisms used by plants to minimize pathogen attack.

By combining $\mathrm{R}$ and $A v r$ gene expression in a single plant genotype, it is possible to engineer a 'trigger' for HR, but constitutive expression of both $\mathrm{R}$ and Avr in the same transgenic plant will cause a whole plant-induced hypersensitivity and results in plant death. Thus, the expression of this two-component system must be tightly regulated (Hammond-Kosack and Kanyuka, 2007). The desired phenotype can be obtained by using synthetic promoters designed only to be pathogen inducible and ensures precise localization of defense activation (Rushton et al., 2002). This strategy has been well tested using the $R$-Avr gene combination of tomato $C f 9$ and C. fulvum Avr9 and found to be highly promising for controlling multiple pathogens (Hammond-Kosack and Kanyuka, 2007).

Most of the R genes exhibit high recognition specificity with elicitors from pathogens. This problem can be overcome through single point mutation, which results in auto-activation of R genes. Specific amino acid changes in the NBS or ARC2 domain of various NBS-LRR R genes such as tomato $I 2$, potato $R x$ and flax $L 6$ result in auto-activation and Avr-independent defense signaling. Alternatively, a random in vitro mutagenesis of the domains in the $\mathrm{R}$ gene sequence can also result in pathogen recognition and subsequent recognition specificities of the selected gene variants. A subset of the mutant $R x$ gene sequences in potato was shown to confer resistance against the original viral strain, as well as additional potato virus X strains, and a second distantly related virus species (Farnham and Baulcombe, 2006).

\section{CONCLUDING REMARKS}

Diseases are the major limiting factor for plant growth and productions. They require dominant or semi-dominant resistance $(\mathrm{R})$ gene alleles to specifically recognize the infecting pathogens. With the isolation of more and more plant NBS-LRR class R genes, immense opportunities are now unfolding in understanding their characteristics and resistance signaling pathways for effective defense responses. However, we are still in the dark about various characteristics of NBS-LRRs. For example, what is the three-dimensional structure of LRR domains in different $\mathrm{R}$ proteins? What is the nature of quantitative disease resistance response? What is the nature of defense response against pathogens feeding on dead tissues? What are the signals responsible for controlling plant development and activation of basal and $\mathrm{R}$ protein defense response? and so on.

Cloning, characterization and sequencing of new plant and pathogen genomes will help us to predict the nature of host-pathogen interactions through the use of modern biotechniques and bioinformatic tools. Moreover, detail knowledge about the events occurring 
in different cellular compartments during host-pathogen interaction can determine the potential points of pathogen weakness and strength of host resistance and create new avenues for sustainable disease control. The complete removal of diseases from plants is not practically possible. Many pathogens continue to evolve due to constraints imposed upon them by new agricultural practices. Therefore, protecting plants through germplasm modification can be a simple solution to disease control. Greater knowledge and insights into the activities of NBSLRR proteins, avr proteins and the subsequent defense signaling networks will be very helpful in designing new disease control strategies.

\section{ACKNOWLEDGMENTS}

The authors are grateful to Prof. Manoj Ranjan Nayak, President of the Siksha O Anusandhan University, for his encouragement and support.

\section{REFERENCES}

Ade J, DeYoung BJ, Golstein C and Innes RW (2007). Indirect activation of a plant nucleotide binding site-leucine-rich repeat protein by a bacterial protease. Proc. Natl. Acad. Sci. U. S. A. 104: 2531-2536.

Asai T, Tena G, Plotnikova J, Willmann MR, et al. (2002). MAP kinase signalling cascade in Arabidopsis innate immunity. Nature 415: 977-983.

Azevedo C, Sadanandom A, Kitagawa K, Freialdenhoven A, et al. (2002). The RAR1 interactor SGT1, an essential component of R gene-triggered disease resistance. Science 295: 2073-2076.

Bai J, Pennill LA, Ning J, Lee SW, et al. (2002). Diversity in nucleotide binding site-leucine-rich repeat genes in cereals. Genome Res. 12: 1871-1884.

Burkhard P, Stetefeld J and Strelkov SV (2001). Coiled coils: a highly versatile protein folding motif. Trends Cell Biol. 11: $82-88$.

Dangl JL and Jones JD (2001). Plant pathogens and integrated defence responses to infection. Nature 411: 826-833.

Deslandes L, Olivier J, Peeters N, Feng DX, et al. (2003). Physical interaction between RRS1-R, a protein conferring resistance to bacterial wilt, and PopP2, a type III effector targeted to the plant nucleus. Proc. Natl. Acad. Sci. U. S. A. 100: 8024-8029.

Dodds PN, Lawrence GJ and Ellis JG (2001). Six amino acid charges confined to the leucine-rich repeat $\beta$-strand/ $\beta$-turn motif determine the difference between the $P$ and $P 2$ rust resistance specificities in flax. Plant Cell 13: 163-178.

Fan W and Dong X (2002). In vivo interaction between NPR1 and transcription factor TGA2 leads to salicylic acidmediated gene activation in Arabidopsis. Plant Cell 14: 1377-1389.

Farnham G and Baulcombe DC (2006). Artificial evolution extends the spectrum of viruses that are targeted by a diseaseresistance gene from potato. Proc. Natl. Acad. Sci. U. S. A. 103: 18828-18833.

Feys BJ and Parker JE (2000). Interplay of signaling pathways in plant disease resistance. Trends Genet. 16: 449-455.

Flor H (1971). Current status of the gene-for-gene concept. Annu. Rev. Phytopathol. 9: 275-296.

Fourmann M, Chariot F, Froger N, Delourme R, et al. (2001). Expression, mapping, and genetic variability of Brassica napus disease resistance gene analogues. Genome 44: 1083-1099.

Frye CA, Tang D and Innes RW (2001). Negative regulation of defense responses in plants by a conserved MAPKK kinase. Proc. Natl. Acad. Sci. U. S. A. 98: 373-378.

Glazebrook J (2001). Genes controlling expression of defense responses in Arabidopsis-2001 status. Curr. Opin. Plant Biol. 4: 301-308.

Graham MA, Marek LF, Lohnes D, Cregan P, et al. (2000). Expression and genome organization of resistance gene analogs in soybean. Genome 43: 86-93.

Hammond-Kosack KE and Jones JD (1997). Plant disease resistance genes. Annu. Rev. Plant Physiol. Plant Mol. Biol. 48: 575-607.

Hammond-Kosack KE and Kanyuka K (2007). Resistance genes (R genes) in plants. Encycl. Life Sci. 40: 1-21.

Hulbert SH, Webb CA, Smith SM and Sun Q (2001). Resistance gene complexes: evolution and utilization. Annu. Rev. Phytopathol. 39: 285-312.

Jia Y, McAdams SA, Bryan GT, Hershey HP, et al. (2000). Direct interaction of resistance gene and avirulence gene 
products confers rice blast resistance. EMBO J. 19: 4004-4014.

Jones DA and Jones JDG (1997). The role of leucine rich repeat proteins in plant defenses. Adv. Bot. Res. 24: 89-167.

Kanazin V, Marek LF and Shoemaker RC (1996). Resistance gene analogs are conserved and clustered in soybean. Proc. Natl. Acad. Sci. U. S. A. 93: 11746-11750.

Keen NT (1990). Gene-for-gene complementarity in plant-pathogen interactions. Annu. Rev. Genet. 24: 447-463.

Kobe B and Kajava AV (2001). The leucine-rich repeat as a protein recognition motif. Curr. Opin. Struct. Biol. 11: 725732.

Kohler A, Rinaldi C, Duplessis S, Baucher M, et al. (2008). Genome-wide identification of NBS resistance genes in Populus trichocarpa. Plant Mol. Biol. 66: 619-636.

Mackey D, Holt BF III, Wiig A and Dangl JL (2002). RIN4 interacts with Pseudomonas syringae type III effector molecules and is required for RPM1-mediated resistance in Arabidopsis. Cell 108: 743-754.

Mago R, Nair S and Mohan M (1999). Resistance gene analogues from rice: cloning, sequencing and mapping. Theor. Appl. Genet. 99: 50-57.

Maldonado AM, Doemer P, Dixon RA, Lamb CJ, et al. (2002). A putative lipid transfer protein involved in systemic resistance signalling in Arabidopsis. Nature 419: 399-403.

McCallum CM, Comai L, Greene EA and Henikoff S (2000). Targeting induced local lesions IN genomes (TILLING) for plant functional genomics. Plant Physiol. 123: 439-442.

McDowell JM and Woffenden BJ (2003). Plant disease resistance genes: recent insights and potential applications. Trends Biotechnol. 21: 178-183.

Mes JJ, van Doorn AA, Wijbrandi J, Simons G, et al. (2000). Expression of the Fusarium resistance gene I-2 colocalizes with the site of fungal containment. Plant J. 23: 183-193.

Meyers BC, Dickerman AW, Michelmore RW, Sivaramakrishnan S, et al. (1999). Plant disease resistance genes encode members of an ancient and diverse protein family within the nucleotide-binding superfamily. Plant J. 20: 317-332.

Meyers BC, Kozik A, Griego A, Kuang H, et al. (2003). Genome-wide analysis of NBS-LRR-encoding genes in Arabidopsis. Plant Cell 15: 809-834.

Meyers BC, Kaushik S and Nandety RS (2005). Evolving disease resistance genes. Curr. Opin. Plant Biol. 8: 129-134.

Mun JH, Kwon SJ, Yang TJ, Seol YJ, et al. (2009). Genome-wide comparative analysis of the Brassica rapa gene space reveals genome shrinkage and differential loss of duplicated genes after whole genome triplication. Genome Biol. 10: R111.

Muskett PR, Kahn K, Austin MJ, Moisan LJ, et al. (2002). Arabidopsis RAR1 exerts rate-limiting control of R genemediated defenses against multiple pathogens. Plant Cell 14: 979-992.

Nair RA and Thomas G (2007). Isolation, characterization and expression studies of resistance gene candidates (RGCs) from Zingiber spp. Theor. Appl. Genet. 116: 123-134.

Pan Q, Wendel J and Fluhr R (2000). Divergent evolution of plant NBS-LRR resistance gene homologues in dicot and cereal genomes. J. Mol. Evol. 50: 203-213.

Parker JE, Coleman MJ, Szabo V, Frost LN, et al. (1997). The Arabidopsis downy mildew resistance gene RPP5 shares similarity to the toll and interleukin-1 receptors with N and L6. Plant Cell 9: 879-894.

Petersen M, Brodersen P, Naested H, Andreasson E, et al. (2000). Arabidopsis map kinase 4 negatively regulates systemic acquired resistance. Cell 103: 1111-1120.

Rushton PJ, Reinstadler A, Lipka V, Lippok B, et al. (2002). Synthetic plant promoters containing defined regulatory elements provide novel insights into pathogen- and wound-induced signaling. Plant Cell 14: 749-762.

Sharma TR, Das A, Kumar SP and Lodha ML (2009). Resistance gene analogues as a tool for rapid identification and cloning of disease resistance genes in plants - a review. J. Plant Biochem. Biotechnol. 18: 1-11.

Tai TH, Dahlbeck D, Clark ET, Gajiwala P, et al. (1999). Expression of the $B s 2$ pepper gene confers resistance to bacterial spot disease in tomato. Proc. Natl. Acad. Sci. U. S. A. 96: 14153-14158.

Tameling WI, Elzinga SD, Darmin PS, Vossen JH, et al. (2002). The tomato R gene products $I-2$ and $M I-I$ are functional ATP binding proteins with ATPase activity. Plant Cell 14: 2929-2939.

Thilmony RL, Chen Z, Bressan RA and Martin GB (1995). Expression of the tomato Pto gene in tobacco enhances resistance to Pseudomonas syringae pv. tabaci expressing avrPto. Plant Cell 7: 1529-1536.

Thomma BP, Penninckx IA, Broekaert WF and Cammue BP (2001). The complexity of disease signaling in Arabidopsis. Curr. Opin. Immunol. 13: 63-68.

Torii KU (2004). Lucine-rich repeat receptor kinase in plants: structure, function, and signal transduction pathways. Inter. Rev. Cytol. 234: 1-46.

Traut TW (1994). The functions and consensus motifs of nine types of peptide segments that form different types of nucleotide-binding sites. Eur. J. Biochem. 222: 9-19. 
Van der Biezen EA and Jones JD (1998). The NB-ARC domain: a novel signalling motif shared by plant resistance gene products and regulators of cell death in animals. Curr. Biol. 8: R226-R227.

Xiao S, Charoenwattana P, Holcombe L and Turner G (2003). The Arabidopsis genes RPW8.1 and RPW8.2 confer induced resistance to powdery mildew diseases in tobaccao. Mol. Plant Microbe Interact. 16: 289-294.

Xu Y, Tao X, Shen B, Horng T, et al. (2000). Structural basis for signal transduction by the Toll/interleukin-1 receptor domains. Nature 408: 111-115.

Yi H and Richard ES (2007). A cluster of disease resistance gene in Arabidopsis is coordinately regulated by transcriptional activation and RNA silencing. Plant Cell 19: 2929-2939.

Yoder MD, Keen NT and Jurnak F (1993). New domain motif: the structure of pectate lyase C, a secreted plant virulence factor. Science 260: 1503-1507.

Yoshimura S, Yamanouchi U, Katayose Y, Toki S, et al. (1998). Expression of Xa1, a bacterial blight-resistance gene in rice, is induced by bacterial inoculation. Proc. Natl. Acad. Sci. U. S. A. 95: 1663-1668.

Zhang R, Cai H, Fatima N, Buczko E, et al. (1995). Functional glycosylation sites of the rat luteinizing hormone receptor required for ligand binding. J. Biol. Chem. 270: 21722-21728.

Zhou T, Wang Y, Chen JQ, Araki H, et al. (2004). Genome-wide identification of NBS genes in japonica rice reveals significant expansion of divergent non-TIR NBS-LRR genes. Mol. Genet. Genom. 271: 402-415. 